\title{
Light-harvesting antenna complexes in the moss Physcomitrella patens: implications for the evolutionary transition from green algae to land plants
}

Masakazu Iwai ${ }^{1,2}$ and Makio Yokono ${ }^{3}$

${ }^{1}$ Department of Plant and Microbial Biology, University of California, Berkeley, CA 94720-3102, USA,

${ }^{2}$ Molecular Biophysics and Integrative Bioimaging Division, Lawrence Berkeley National Laboratory, Berkeley, CA 94720, USA,

${ }^{3}$ Institute of Low Temperature Science, Hokkaido University, Sapporo 060-0819, Japan.

Corresponding author: Iwai, Masakazu (miwai@berkeley.edu) 


\section{Abstract}

Plants have successfully adapted to a vast range of terrestrial environments during their evolution. To elucidate the evolutionary transition of light-harvesting antenna proteins from green algae to land plants, the moss Physcomitrella patens is ideally placed basally among land plants. Compared to the genomes of green algae and land plants, the P. patens genome codes for more diverse and redundant light-harvesting antenna proteins. It also encodes Lhcb9, which has characteristics not found in other light-harvesting antenna proteins. The unique complement of light-harvesting antenna proteins in $P$. patens appears to facilitate protein interactions that include those lost in both green algae and land plants with regard to stromal electron transport pathways and photoprotection mechanisms. This review will highlight unique characteristics of the $P$. patens light-harvesting antenna system and the resulting implications about the evolutionary transition during plant terrestrialization.

\section{Introduction}

The extant terrestrial flora displays remarkably diverse characteristics and has succeeded in dominating incredibly varied environments. During the evolution of green plants, the transition from aquatic to terrestrial environments was a key process involving a series of changes conferring the ability to cope with terrestrial conditions including exposure to dry air, gravity, atmospheric $\mathrm{CO}_{2}$, and light. To address fundamental questions related to plant terrestrialization, bryophytes (liverworts, mosses, and hornworts) are ideally positioned basally among the extant land plants. The moss Physcomitrella patens has been developed as a model organism [1], and its genome data have provided new knowledge about how the early land plant lineage would have facilitated the adaptation to terrestrial environment $[2,3]$.

As photosynthesis is the major source of energy for photoautotrophic organisms, 
the change in light regime during plant terrestrialization must have been a substantial environmental stress [4]. Although the fundamental architecture of the photosynthetic reaction centers is highly conserved among photoautotrophic organisms, the light-harvesting antenna proteins are quite diverse, which is suggested to reflect the changes essential for adaptation to different environments [5]. Interestingly, $P$. patens possesses light-harvesting antenna proteins even more diverse than those found in either green algae or land plants [6]. P. patens also has its own unique light-harvesting antenna protein, Lhcb9 [7]. Furthermore, the light energy dissipation mechanism, so-called non-photochemical quenching (NPQ), that is activated under excess light conditions is also operated by both green algal- and land plant-type proteins in P. patens [8]. Recent progress

in elucidating the molecular mechanisms of NPQ induction in P. patens has been covered in detail elsewhere [9]. Here, we focus on what the unique characteristics of the $P$. patens light-harvesting antenna system imply about the evolutionary transition from green algae to land plants.

\section{The light-harvesting antenna system is redundantly diversified in $P$. patens}

Light-harvesting complex (LHC) proteins are a protein superfamily that bind chlorophylls (Chls) and carotenoids to facilitate the absorption of light energy and the transfer of excitation energy to the reaction centers of photosystems I (PSI) and II (PSII). LHC genes are generally classified into two groups, Lhca and Lhcb, which encode LHC proteins for PSI (LHCI) and PSII (LHCII), respectively [10]. In the green alga Chlamydomonas reinhardtii and the land plant Arabidopsis thaliana, there are at least 9 and 6 Lhca genes, respectively (Table 1). In addition, there are at least 12 and 15 Lhcb genes in C. reinhardtii and $A$. thaliana, respectively. LHCII proteins are further categorized into two groups, the major and 
minor LHClls, which normally exist as trimers or monomers, respectively. In $A$. thaliana, Lhcb1, Lhcb2, and Lhcb3 encode major LHClls, and Lhcb4, Lhcb5, and Lhcb6 encode minor LHClls [10]. In green algae, the genes encoding major LHCIls are designated as Lhcbm ("m" for major) and have relatively low amino acid sequence similarity to $A$. thaliana Lhcb1 (AtLhcb1; “At” for A. thaliana, hereafter), AtLhcb2, and AtLhcb3 [11,12].

The $P$. patens genome contains more LHC genes with greater redundancy compared to those of both the green alga $C$. reinhardtii and the land plant $A$. thaliana (Table 1). For example, the orthologs of AtLhca1 and AtLhca2 are encoded by 3 and 5 paralogous genes in P. patens, respectively (Figure 1). The homologs of AtLhca3 seem to have arisen prior to the divergence of green algal lineages [12], and both $C$. reinhardtii and $A$. thaliana have one Lhca3 gene. However, the P. patens genome contains four paralogs for Lhca3. The Lhcbm proteins in $P$. patens clearly show more diversity and redundancy compared to those in the other species, and some of their amino acid sequences are almost identical even though the loci are different (the genes encoding major LHCII in P. patens are designated as Lhcbm as well [6]). There are at least four different isoforms of Lhcb5 in $P$. patens, although most other organisms contain only one [2], except for the recently sequenced plants soybean and Kalanchoe laxiflora [v1.1, Department of Energy-The Joint Genome Institute (DOE-JGI), http://phytozome.jgi.doe.gov/], which is a model plant well-adapted to dry growth conditions, [13]. Not only LHC genes, but other genes, for example those related to housekeeping and metabolic pathways, are highly abundant in the $P$. patens genome, which is therefore suggested to arise from genome-wide duplication events in the ancestral lineage of mosses $[3,14,15]$.

In addition to the diversified LHC proteins, the $P$. patens genome shows intriguing composition of the light-harvesting antenna system. For example, the ortholog of AtLhca4 is 
missing in $P$. patens, although it appears to be important for stabilizing the PSI-LHCI structure in $A$. thaliana [16], resulting in a blue-shift in the low-temperature fluorescence emission of the P. patens PSI-LHCI supercomplex [6]. P. patens contains an ortholog of AtLhca5, but not of AtLhca6, both of which are essential for the formation of the supercomplex comprising chloroplast type I NADH dehydrogenase (NDH) and PSI in $A$. thaliana [17], and in fact only a partial NDH-PSI supercomplex is assembled in P. patens [18]. Another bryophyte, the liverwort Marchantia polymorpha, lacks orthologs of both AtLhca5 and AtLhca6, and the NDH-PSI supercomplex has not been detected in that species [19]. The different PSI light-harvesting antenna system found in P. patens, in addition to some exceptional characteristics of PSI subunits (e.g., subunit F is a primitive type, three different isoforms of subunit $\mathrm{K}$ are present, the cyanobacterial-type subunit $\mathrm{M}$ is present, and subunit $\mathrm{N}$ is missing [20]), may contribute to a unique set of protein-protein interactions involved with PSI assembly in the moss.

Along with the Lhcbm genes encoding LHCII proteins, P. patens also has an ortholog of AtLhcb3, while $C$. reinhardtii does not, suggesting its acquisition during plant terrestrialization [6] (however, Lhcb3 appears to have been lost during the divergence of Pinaceae and Gnetales [21]). The orthologs of three minor LHCIl proteins (Lhcb4, Lhcb5, and Lhcb6) in A. thaliana are also found in P. patens [6]. It appears that none of the P. patens Lhcb4 (PpLhcb4; "Pp" for P. patens, hereafter) isoforms are related to AtLhcb4.3 [6], which is found only in dicots and is now classified as Lhcb8 due to its distinct function from AtLhcb4.1 and AtLhcb4.2 [22]. Further, as the ortholog of AtLhcb6 has not been found in green algae so far [11,12], the presence of an AtLhcb6 ortholog in $P$. patens suggests its uniqueness to land plants, although it is absent in Pinaceae and Gnetales, similar to the case of Lhcb3 [21]. In addition to major and minor LHClls, the ortholog of AtLhcb7, which is rarely expressed 
[22], is present in $P$. patens [6].

It is of interest that $P$. patens has maintained a more diversified set of LHC proteins than has $A$. thaliana, which has also adapted to nonaquatic conditions. Given its lack of anatomical adaptation to terrestrial conditions, with a simplified morphology and poikilohydry, the highly diversified LHC genes in P. patens might confer the robustness of the light-harvesting antenna system required for the unique physiological conditions encountered during the terrestrialization of their ancestral lineage.

\section{Lhcb9 is a unique Lhcb protein found in $P$. patens}

Intriguingly, a novel Lhcb gene Lhcb9 is found in P. patens [6]. According to Phytozome v.11.0, a Lhcb9-like protein appears to be present in the bog moss Sphagnum fallax (v0.5, DOE-JGI), but no ortholog of Lhcb9 is found in other sequenced taxa so far. Based on its sequence, Lhcb9 appears to belong to the group of LHCII proteins and most likely exists as a monomer $[6,7]$. However, one of the unique features of Lhcb9 is that it binds Chl pigments that show fluorescence emission longer than $\sim 680 \mathrm{~nm}$, the typical fluorescence emission maximum of LHCII [7]. Analyses using the reconstituted Lhcb9 complex suggest that an asparagine, instead of a histidine, serves as the central ligand for $\mathrm{Chl} 603$, which is a characteristic observed in AtLhca3 and AtLhca4 [23] but not in Lhcb proteins, and is responsible for the red-shifted fluorescence emission [7]. Although the degree of red-shift of Chl 603 is smaller than that of "red Chls" observed in the PSI light-harvesting antenna system [24], Lhcb9 is the only Lhcb protein showing this feature.

Two studies have shown contradictory results regarding the association of Lhcb9 with PSII [7], as might be expected for a protein related to Lhcb family members, or with PSI [25], which may arise from the different growth conditions used, leading to the different 
localization of Lhcb9. The exact localization of Lhcb9 is thus unclear without further analyses. One study found that the association of Lhcb9 with PSI results in the presence of two different sizes of PSI-LHCI supercomplexes in P. patens thylakoid membranes [25]. Interestingly, the smaller and larger PSI-LHCI supercomplexes have similar sizes as PSI-LHCI supercomplexes in $A$. thaliana and $C$. reinhardtii, respectively (Figure 2). In the $A$. thaliana PSI-LHCI supercomplex, four LHCI proteins are aligned laterally into a crescent shape (the so-called LHCI belt) and positioned at the PsaG/F/J/K side of the PSI core $[16,26]$. By contrast, the $C$. reinhardtii PSI-LHCI supercomplex harbors up to nine $\mathrm{LHCl}$ proteins (CrLhca1-9; "Cr" for $C$. reinhardtii, hereafter), forming a double crescent shape located at the PsaG/F/J/K side of the PSI core [27]. In a $P$. patens Lhcb9 knockout line, the larger PSI-LHCI supercomplex is completely missing, suggesting that Lhcb9 could function as a linker between the smaller PSI-LHCI supercomplex and the additional antenna that forms the larger supercomplex [25]. Although the structural organization of this larger PSI-LHCI supercomplex in P. patens still needs to be resolved, having the two different sizes of PSI-LHCl supercomplexes that correspond to the ones in A. thaliana and C. reinhardtii might reflect the evolutionary demand for the transition during plant terrestrialization.

Another curious feature about Lhcb9 is that its most similar protein outside of mosses is Lhcbm5 from C. reinhardtii [25] (the moss $S$. fallax contains most similar Lhcb9). All other Lhca and Lhcb proteins in $P$. patens are most similar to the ones in $A$. thaliana (except for PpLhcb7, which is more similar to the one in Populus trichocarpa). Only Lhcb9 appears to be distantly related to land plant LHC proteins. Phylogenetic analyses also suggest that Lhcb9 was derived prior to the divergence from green algal LHC (i.e., is orthologous to CrLhcbm5), while all the other LHC proteins appear to have evolved after that [25] (Figure 3). The liverwort M. polymorpha does not seem to contain the ortholog of Lhcb9 
(v3.1, DOE-JGI), highlighting the uniqueness of this protein even within the bryophyte lineage. One possible explanation for this inconsistency is that the ancestral LHC protein for Lhcb9 was introduced to $P$. patens genome by horizontal gene transfer (HGT) during the evolution. Intriguingly, the most similar LHC to Lhcb9, CrLhcbm5 is also found to be associated with the PSI-LHCI supercomplex in C. reinhardtii [28]. The shared ability to bind with PSI might be derived from their common ancestral LHC protein present in the earlier green algal lineage, which evolved into CrLhcbm5 in the succeeding $C$. reinhardtii, while the one horizontally transferred to the early moss lineage evolved into Lhcb9 in P. patens. As HGT is essential for the transition during plant terrestrialization [29], it is hypothesized that the Lhcb9-mediated, larger PSI-LHCI supercomplex might have promoted survival under certain environmental conditions for the ancestral lineage of mosses [25].

\section{Multiple chloroplast stromal electron flow pathways operate in $P$. patens}

LHCII proteins not only transfer light energy to photosystems but dissipate the energy as heat to prevent photodamage under excess light conditions via the energy-dependent type of NPQ, or qE (reviewed in [30]). The molecular details of the qE mechanism are still debated, but the development of the transmembrane $\mathrm{pH}$ gradient across thylakoid membrane $(\Delta \mathrm{pH})$ is known to be the trigger. The $\Delta \mathrm{pH}$ is generated by several factors related to linear and cyclic electron flow in different, alternative pathways (reviewed in [31]). In linear electron flow, water oxidation by PSIl and the proton translocation by cytochrome $b_{6} f(\mathrm{Q}$ cycle) generate $\Delta \mathrm{pH}$. In cyclic electron flow, the electrons generated at the stromal side of PSI are transferred via ferredoxin back to the PGR5/PGRL1 complex in thylakoid membranes, which reduces plastoquinone, inducing the $\mathrm{Q}$ cycle and a buildup of $\Delta \mathrm{pH}$, in both $C$. reinhardtii [32] and $A$. thaliana $[33,34]$. $A$. thaliana has an alternative pathway that is 
dependent on PSI-NDH complex (reviewed in [35]). In C. reinhardtii, the formation of a supercomplex including PSI-LHCI and cytochrome $b_{6} f$, which also involves the association of ferredoxin-NADPH oxidoreductase, PGRL1, $\mathrm{Ca}^{2+}$ sensor protein, and anaerobic response 1 protein, is shown to mediate cyclic electron flow [36-38].

In P. patens, PGR5/PGRL1-dependent cyclic electron flow appears to operate, based on analyses using the pgrl1 knockout mutant [39]. Interestingly, it is also suggested that PGRL1 is required for survival under high light and anoxic conditions in $C$. reinhardtii and $P$. patens, and thus might have been essential for plant terrestrialization [39]. The NDH complex is absent in green algae and some gymnosperms but present in $P$. patens. The association of the NDH complex with the PSI-LHCI supercomplex enhances cyclic electron transport in $A$. thaliana, although only partial supercomplex formation is observed in $P$. patens, which might be due to the lack of PpLhca6 [18]. Alternatively, $\Delta \mathrm{pH}$ can be developed through photoreduction of oxygen in the chloroplast stroma via flavodiiron (Flv) proteins, which are found in cyanobacteria, green algae, mosses, and gymnosperms but are absent from flowering plants [40]. It has been shown that Flv proteins in $P$. patens play a substantial role as electron sinks, which prevents photodamage in PSI, and are important for survival under fluctuating light conditions [41]. Although Flv proteins were lost in flowering plants during evolution, PpFlv proteins are functional when expressed in $A$. thaliana, which leaves us with the question of why they were lost [42]. P. patens thus operates multiple ways of electron transport and generating $\Delta \mathrm{pH}$, which must have been physiologically essential for the evolution of moss lineage and might reflect an evolutionary demand during the transition to terrestrial environments.

\section{Mechanisms for excess light energy dissipation are independently induced by}




\section{LHCSR and PsbS in P. patens}

In plants, the buildup of $\Delta \mathrm{pH}$ activates the thylakoid lumenal enzyme violaxanthin deepoxidase, converting violaxanthin to zeaxanthin, accumulation of which is essential for the induction of qE [43]. In C. reinhardtii, high light stress-related LHC (LHCSR [44]; originally known as L1818) protein is protonated on its lumenal side upon buildup of $\Delta \mathrm{pH}$ [45], which enhances the induction of qE within the PSII-LHCII-LHCSR supercomplex [46]. PSII subunit S (PsbS) was recently shown to be expressed transiently during the activation of qE and stabilize the accumulation of LHCSR, implying a pivotal role for PsbS in qE induction in C. reinhardtii [47]. In land plants, PsbS plays a key role in the induction of qE by sensing $\Delta \mathrm{pH}$ [48] and reorganizing LHCII around PSII, while LHCSR was lost during the evolution of land plants (reviewed in [49]).

Interestingly, LHCSR is conserved in P. patens and S. fallax but appears to be lost in the liverwort M. polymorpha (v3.1) and the lycophyte Selaginella moellendorffii (v1.0, DOE-JGI). In P. patens, LHCSR is independently functional in addition to PsbS to induce qE $[8,50,51]$. Unlike its orthologs in C. reinhardtii [45], the qE activity of PpLHCSR is largely reliant on the accumulation of zeaxanthin [52]. The two PpLHCSR isoforms, LHCSR1 and LHCSR2, are more responsible for quenching under high light or low temperature, respectively [53]. Also, unlike CrPsbS [47], PpPsbS is expressed under normal conditions and involved in the induction of qE [8]. Intriguingly, transient expression measurements of NPQ in Nicotiana benthamiana show that PpPsbS is able to induce faster and greater quenching than AtPsbS and CrPsbS [54]. A similar approach revealed an exceptional feature of PpLHCSR1-it lacks $\mathrm{Chl} b$, which is usually required by LHC proteins for folding [55]. Surprisingly, the localizations of PpLHCSR and PpPsbS appear to be exclusive of each other in $P$. patens thylakoid membranes, where the former is found mostly in grana margin 
and stroma lamellae, and the latter is localized only in grana [56]. It has been suggested that PpLHCSR can associate with both PSI and PSII to activate qE under excess light conditions [56], while PpPsbS interacts with the moderately-bound major LHCII in the PSII supercomplex [57], as similarly suggested in $A$. thaliana [30]. The dual mechanisms of PpLHCSR and PpPsbS to induce qE clearly imply the requirement for conquering newly experienced light environments during plant terrestrialization.

\section{Concluding remarks}

After at least 450 million years [58], the mosses have flourished in almost all terrestrial environments. Being poikilohydric, the characteristics that mosses derived from green algal-lineage genes might have been effective for acclimating to semiaquatic conditions, while the whole-genome duplication and HGT might have led them to acquire the characteristics required for survival in terrestrial conditions. Here, we have discussed only the unique characteristics pertaining to the light-harvesting antenna system in $P$. patens. Together with knowledge about other physiological features and mechanisms, which have also been substantially investigated using this moss, we have an avenue to a more complete understanding of how the light-harvesting antenna system evolved strategically during plant terrestrialization. Further, comparative genomics and phylogenomics analyses using other organisms positioned evolutionarily between green algae and land plants, such as freshwater green algae (charophytes) and liverworts, in relation to the paleophysiological aspects of each species should provide fascinating insights into the evolutionary dynamics of light-harvesting antenna systems.

\section{Acknowledgments}


M.I. was supported by the U.S. Department of Energy, Office of Science, Basic Energy Sciences, Chemical Sciences, Geosciences, and Biosciences Division under field work proposal SISGRKN. M.Y. was supported by Core Research for Evolutional Science and Technology, Japan Science and Technology Agency. We apologize to colleagues whose works we could not cite and/or discuss extensively due to space constraints. 


\section{Table 1. Phenotype comparison among C. reinhardtii, P. patens, and $A$.}

\section{thaliana.}

\begin{tabular}{|c|c|c|c|c|c|c|c|c|c|c|}
\hline LHCl & Lhca1 & Lhca2 & Lhca3 & Lhca4 & \multicolumn{2}{|c|}{ Lhca5 } & Lhca6 & Lhca7 & Lhca8 & Lhca9 \\
\hline C. reinhardtii & 1 & 1 & 1 & 1 & 1 & & 1 & 1 & 1 & 1 \\
\hline P. patens & 3 & 5 & 4 & 0 & 1 & & 0 & 0 & 0 & 0 \\
\hline A. thaliana & 1 & 1 & 1 & 1 & 1 & & 1 & 0 & 0 & 0 \\
\hline LHCII & Lhcb1 & Lhcb2 & Lhcb3 & Lhcbm & Lhcb4 & Lhcb5 & Lhcb6 & Lhcb7 & Lhcb8 & Lhcb9 \\
\hline C. reinhardtii & 0 & 0 & 0 & 9 & 1 & 1 & 0 & 1 & 0 & 0 \\
\hline P. patens & 0 & 0 & 1 & 14 & 2 & 4 & 2 & 1 & 0 & 2 \\
\hline A. thaliana & 5 & 3 & 1 & 0 & 2 & 1 & 1 & 1 & 1 & 0 \\
\hline
\end{tabular}

Number indicates the number of orthologous Lhca and Lhcb genes identified in C. reinhardtii genome (v5.5, DOE-JGl), $P$. patens genome (v3.3, DOE-JGI), and $A$. thaliana genome (The Arabidopsis Information Resource annotation release 10). Lhcb8 is previously known as Lhcb4.3 in A. thaliana [22].

\begin{tabular}{|c|c|c|c|c|c|c|}
\hline & \multicolumn{4}{|c|}{ Stromal electron flow pathways } & \multicolumn{2}{|c|}{$q E$} \\
\hline & PGR5/PGRL1 & PSI-NDH & PSI-Cyt $b_{6} f$ & Flv & LHCSR & PsbS \\
\hline C. reinhardtii & Yes & No & Yes & Yes & Yes & Transient \\
\hline P. patens & Yes & Partial & TBD & Yes & Yes & Yes \\
\hline A. thaliana & Yes & Yes & TBD & No & No & Yes \\
\hline
\end{tabular}

Three protein complexes operating cyclic electron flow exist-PGR5/PGRL1 complex (PGR5/PGRL1), PSI-NDH complex (PSI-NDH), and PSI-Cyt $b_{6} f$ complex (PSI-Cyt $b_{6} f$ ). There is also flavodiiron protein (Flv)-dependent pathway exists as an alternative pathway [31,35]. TBD, to be determined. The partial PSI-NDH complex is observed in P. patens (Partial) [18]. Energy-dependent non-photochemical quenching (qE) is operated by LHCSR and/or PsbS. PsbS is expressed transiently during the activation of $\mathrm{qE}$ in $C$. reinhardtii (Transient) [47]. 


\section{Figure 1}

Phylogenetic diversity of LHC proteins. The analysis includes amino acid sequences from LHC proteins found in $A$. thaliana (At), P. patens $(\mathrm{Pp})$, and $C$. reinhardtii $(\mathrm{Cr})$. The sequences for $A$. thaliana were retrieved from The Arabidopsis Information Resource, and those for $P$. patens and C. reinhardtii were retrieved from JGI (v3.3 and v5.5, respectively). The positions of Lhca and Lhcb proteins in PSI and PSII supercomplexes, respectively, are indicated. The stable structures for PSII-LHCII and PSI-LHCI are drawn according to Protein Data Bank (3WU2, 2BHW, 3PL9, 3JCU, and 4XK8).

\section{Figure 2}

Density gradient analysis of solubilized thylakoid membrane proteins. Thylakoid membranes (100 $\mu \mathrm{g} \mathrm{Chl} \mathrm{at} 0.5 \mathrm{mg} \mathrm{Chl} / \mathrm{mL}$ ) isolated from $P$. patens WT and Lhcb9 knockout mutant ( $\Delta$ Lhcb9), A. thaliana Col-0 (WT), and C. reinhardtii $4 \mathrm{~A}^{+}(\mathrm{WT})$ were solubilized with $1 \%$

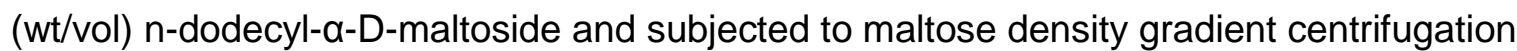
[0.1-1.3 M maltose with $25 \mathrm{mM}$ MES-NaOH (pH 6.5) and 0.03\% (wt/vol) n-dodecyl-a-D-maltoside] at 154,300 $\times g$ (SW 41 Ti rotor, Beckman Coulter) for $24 \mathrm{~h}$ at $4^{\circ} \mathrm{C}$. Arrowheads and arrows indicate the bands corresponding to the smaller and larger PSI-LHCI supercomplexes, respectively. Dots indicate the bands corresponding to PSII-LHCII supercomplexes.

\section{Figure 3}

Phylogenetic tree of Lhcb9 and the most closely related LHC proteins of other photosynthetic eukaryotes. Lhcb9 was used as query sequence for BLAST searches against the genomes of 9 organisms [land plants, Arabidopsis thaliana (Lhcb2.1); bryophytes, Marchantia polymorpha (Mapoly0026s0025), Physcomitrella patens (Lhcb9.1), and Sphagnum fallax (Sphfalx0043s0013); chlorophytes, Chlamydomonas reinhardtii (Lhcbm5) and Volvox carteri (Vocar.0014s0016); prasinophytes, Ostreococcus lucimarinus (XP_001420273) and O. tauri (XP_003081908); red algae, Cyanidioschyzon merolae (CMQ142C); and diatoms, Thalassiosira pseudonana (XP_002295184); In parentheses, the names of LHC protein are indicated, whereas the locus names are provided in case that no 
specific LHC name is annotated.]. The scale bar indicates the expected fraction of amino acids changed. 


\section{References and recommended reading}

Papers of particular interest, published within the period of review, have been highlighted as:

* of special interest

** of outstanding interest

1. Schaefer DG: A new moss genetics: targeted mutagenesis in Physcomitrella patens. Annu Rev Plant Biol 2002, 53:477-501.

2. Rensing SA, Lang D, Zimmer AD, Terry A, Salamov A, Shapiro H, Nishiyama T, Perroud PF, Lindquist EA, Kamisugi Y, et al.: The Physcomitrella genome reveals evolutionary insights into the conquest of land by plants. Science 2008, 319:64-69.

3. Zimmer AD, Lang D, Buchta K, Rombauts S, Nishiyama T, Hasebe M, Van de Peer Y, Rensing SA, Reski R: Reannotation and extended community resources for the genome of the non-seed plant Physcomitrella patens provide insights into the evolution of plant gene structures and functions. BMC Genomics 2013, 14:498.

4. Maberly SC: The fitness of the environments of air and water for photosynthesis, growth, reproduction and dispersal of photoautotrophs: An evolutionary and biogeochemical perspective. Aquatic Botany 2014, 118:4-13.

5. Ganeteg U, Kulheim C, Andersson J, Jansson S: Is each light-harvesting complex protein important for plant fitness? Plant Physiol 2004, 134:502-509.

6. Alboresi A, Caffarri S, Nogue F, Bassi R, Morosinotto T: In silico and biochemical analysis of Physcomitrella patens photosynthetic antenna: identification of subunits which evolved upon land adaptation. PLoS One 2008, 3:e2033.

7. Alboresi A, Gerotto C, Cazzaniga S, Bassi R, Morosinotto T: A red-shifted antenna protein associated with photosystem II in Physcomitrella patens. J Biol Chem 2011, 286:28978-28987.

** This paper shows that Lhcb9, a unique LHC protein in Physcomitrella patens, contains a red-shifted Chl, which is caused by the asparagine ligand for a Chl 603. The data also suggest the association of Lhcb9 with PSII-LHCII supercomplex.

8. Alboresi A, Gerotto C, Giacometti GM, Bassi R, Morosinotto T: Physcomitrella patens mutants affected on heat dissipation clarify the evolution of photoprotection mechanisms 
upon land colonization. Proc Natl Acad Sci U S A 2010, 107:11128-11133.

9. Morosinotto T, Bassi R: Molecular mechanisms for activation of non-photochemical

fluorescence quenching: from unicellular algae to mosses and higher plants. In Non-Photochemical Quenching and Energy Dissipation in Plants, Algae and Cyanobacteria.

Edited by Demmig-Adams B, Garab G, Adams III W, Govindjee: Springer Netherlands; 2014:315-331.

10. Jansson S: A guide to the Lhc genes and their relatives in Arabidopsis. Trends Plant Sci 1999, 4:236-240.

11. Elrad D, Grossman AR: A genome's-eye view of the light-harvesting polypeptides of Chlamydomonas reinhardtii. Curr Genet 2004, 45:61-75.

12. Koziol AG, Borza T, Ishida K, Keeling P, Lee RW, Durnford DG: Tracing the evolution of the light-harvesting antennae in chlorophyll a/b-containing organisms. Plant Physiol 2007, 143:1802-1816.

13. Schmutz J, Cannon SB, Schlueter J, Ma J, Mitros T, Nelson W, Hyten DL, Song Q, Thelen JJ, Cheng J, et al.: Genome sequence of the palaeopolyploid soybean. Nature 2010, 463:178-183.

14. Rensing SA, Ick J, Fawcett JA, Lang D, Zimmer A, Van de Peer Y, Reski R: An ancient genome duplication contributed to the abundance of metabolic genes in the moss Physcomitrella patens. BMC Evol Biol 2007, 7:130.

15. Lang D, Zimmer AD, Rensing SA, Reski R: Exploring plant biodiversity: the Physcomitrella genome and beyond. Trends Plant Sci 2008, 13:542-549.

16. Wientjes E, Oostergetel GT, Jansson S, Boekema EJ, Croce R: The role of Lhca complexes in the supramolecular organization of higher plant photosystem I. J Biol Chem 2009, 284:7803-7810.

17. Peng L, Fukao Y, Fujiwara M, Takami T, Shikanai T: Efficient operation of NAD(P)H dehydrogenase requires supercomplex formation with photosystem I via minor LHCI in Arabidopsis. Plant Cell 2009, 21:3623-3640.

18. Armbruster U, Ruhle T, Kreller R, Strotbek C, Zuhlke J, Tadini L, Blunder T, Hertle AP, Qi Y, Rengstl B, et al.: The photosynthesis affected mutant68-like protein evolved from a PSII assembly factor to mediate assembly of the chloroplast $\mathrm{NAD}(\mathrm{P}) \mathrm{H}$ dehydrogenase 
complex in Arabidopsis. Plant Cell 2013, 25:3926-3943.

19. Ueda M, Kuniyoshi T, Yamamoto H, Sugimoto K, Ishizaki K, Kohchi T, Nishimura Y, Shikanai T:

Composition and physiological function of the chloroplast NADH dehydrogenase-like complex in Marchantia polymorpha. Plant J 2012, 72:683-693.

20. Busch A, Petersen J, Webber-Birungi MT, Powikrowska M, Lassen LM, Naumann-Busch B, Nielsen AZ, Ye J, Boekema EJ, Jensen ON, et al.: Composition and structure of photosystem I in the moss Physcomitrella patens. J Exp Bot 2013, 64:2689-2699.

21. Kouril R, Nosek L, Bartos J, Boekema EJ, llik P: Evolutionary loss of light-harvesting proteins Lhcb6 and Lhcb3 in major land plant groups-break-up of current dogma. New Phytol 2016, 210:808-814.

22. Klimmek F, Sjodin A, Noutsos C, Leister D, Jansson S: Abundantly and rarely expressed Lhc protein genes exhibit distinct regulation patterns in plants. Plant Physiol 2006, 140:793-804.

23. Morosinotto T, Breton J, Bassi R, Croce R: The nature of a chlorophyll ligand in Lhca proteins determines the far red fluorescence emission typical of photosystem I. J Biol Chem 2003, 278:49223-49229.

24. Gobets B, van Grondelle R: Energy transfer and trapping in photosystem I. Biochim Biophys Acta 2001, 1507:80-99.

25. Iwai M, Yokono M, Kono M, Noguchi K, Akimoto S, Nakano A: Light-harvesting complex Lhcb9 confers a green alga-type photosystem I supercomplex to the moss Physcomitrella patens. Nat Plants 2015, 1:14008.

** This paper shows that Lhcb9 is associated with the PSI-LHCI supercomplex, leading to the presence of two different sizes of PSI-LHCI supercomplexes in Physcomitrella patens. Phylogenetic analysis also suggests that the ancestor of $P$. patens might have acquired Lhcb9 through horizontal gene transfer.

26. Boekema EJ, Jensen PE, Schlodder E, van Breemen JF, van Roon H, Scheller HV, Dekker JP: Green plant photosystem I binds light-harvesting complex I on one side of the complex. Biochemistry 2001, 40:1029-1036.

27. Drop B, Webber-Birungi M, Fusetti F, Kouril R, Redding KE, Boekema EJ, Croce R: Photosystem I of Chlamydomonas reinhardtii contains nine light-harvesting complexes (Lhca) 
located on one side of the core. J Biol Chem 2011, 286:44878-44887.

28. Takahashi H, Iwai M, Takahashi Y, Minagawa J: Identification of the mobile light-harvesting complex II polypeptides for state transitions in Chlamydomonas reinhardtii. Proc Natl Acad Sci U S A 2006, 103:477-482.

29. Yue J, Hu X, Sun H, Yang Y, Huang J: Widespread impact of horizontal gene transfer on plant colonization of land. Nat Commun 2012, 3:1152.

30. Ruban AV: Nonphotochemical chlorophyll fluorescence quenching: mechanism and effectiveness in protecting plants from photodamage. Plant Physiol 2016, 170:1903-1916.

31. Strand DD, Kramer DM: Control of non-photochemical exciton quenching by the proton circuit of photosynthesis. In Non-Photochemical Quenching and Energy Dissipation in Plants, Algae and Cyanobacteria. Edited by Demmig-Adams B, Garab G, Adams III W, Govindjee: Springer Netherlands; 2014:387-408.

32. Johnson X, Steinbeck J, Dent RM, Takahashi H, Richaud P, Ozawa S, Houille-Vernes L, Petroutsos D, Rappaport F, Grossman AR, et al.: Proton gradient regulation 5-mediated cyclic electron flow under ATP- or redox-limited conditions: a study of $\triangle A T P a s e$ pgr 5 and $\triangle r b c L$ pgr5 mutants in the green alga Chlamydomonas reinhardtii. Plant Physiol 2014, 165:438-452.

33. Munekage $\mathrm{Y}$, Hojo M, Meurer J, Endo T, Tasaka M, Shikanai T: PGR5 is involved in cyclic electron flow around photosystem I and is essential for photoprotection in Arabidopsis. Cell 2002, 110:361-371.

34. DalCorso G, Pesaresi P, Masiero S, Aseeva E, Schunemann D, Finazzi G, Joliot P, Barbato R, Leister D: A complex containing PGRL1 and PGR5 is involved in the switch between linear and cyclic electron flow in Arabidopsis. Cell 2008, 132:273-285.

35. Peltier G, Aro EM, Shikanai T: NDH-1 and NDH-2 plastoquinone reductases in oxygenic photosynthesis. Annu Rev Plant Biol 2016, 67:55-80.

36. Iwai M, Takizawa K, Tokutsu R, Okamuro A, Takahashi Y, Minagawa J: Isolation of the elusive supercomplex that drives cyclic electron flow in photosynthesis. Nature 2010, 464:1210-1213.

37. Terashima M, Petroutsos D, Hudig M, Tolstygina I, Trompelt K, Gabelein P, Fufezan C, Kudla J, 
Weinl S, Finazzi G, et al.: Calcium-dependent regulation of cyclic photosynthetic electron transfer by a CAS, ANR1, and PGRL1 complex. Proc Natl Acad Sci U S A 2012, 109:17717-17722.

38. Takahashi H, Clowez S, Wollman FA, Vallon O, Rappaport F: Cyclic electron flow is redox-controlled but independent of state transition. Nat Commun 2013, 4:1954.

39. Kukuczka B, Magneschi L, Petroutsos D, Steinbeck J, Bald T, Powikrowska M, Fufezan C, Finazzi G, Hippler M: Proton gradient regulation5-like1-mediated cyclic electron flow is crucial for acclimation to anoxia and complementary to nonphotochemical quenching in stress adaptation. Plant Physiol 2014, 165:1604-1617.

* This paper reveals that PGRL1 and LHCSR3 are important for both Chlamydomonas reinhardtii and Physcomitrella patens to survive under anoxic and high light conditions. The essential role of PGRL1 during plant terrestrialization is also suggested.

40. Allahverdiyeva Y, Isojarvi J, Zhang P, Aro EM: Cyanobacterial oxygenic photosynthesis is protected by flavodiiron proteins. Life 2015, 5:716-743.

41. Gerotto C, Alboresi A, Meneghesso A, Jokel M, Suorsa M, Aro EM, Morosinotto T: Flavodiiron proteins act as safety valve for electrons in Physcomitrella patens. Proc Natl Acad Sci U S A 2016, 113:12322-12327.

* This paper demonstrates a significant role for flavodiiron proteins as an electron sink and in photoprotection under fluctuating light conditions in Physcomitrella patens.

42. Yamamoto $H$, Takahashi S, Badger MR, Shikanai T: Artificial remodelling of alternative electron flow by flavodiiron proteins in Arabidopsis. Nat Plants 2016, 2:16012.

43. Niyogi KK, Grossman AR, Bjorkman O: Arabidopsis mutants define a central role for the xanthophyll cycle in the regulation of photosynthetic energy conversion. Plant Cell 1998, 10:1121-1134.

44. Peers G, Truong TB, Ostendorf E, Busch A, Elrad D, Grossman AR, Hippler M, Niyogi KK: An ancient light-harvesting protein is critical for the regulation of algal photosynthesis. Nature 2009, 462:518-521.

45. Bonente G, Ballottari M, Truong TB, Morosinotto T, Ahn TK, Fleming GR, Niyogi KK, Bassi R: Analysis of LhcSR3, a protein essential for feedback de-excitation in the green alga Chlamydomonas reinhardtii. PLOS Biol 2011, 9:e1000577. 
46. Tokutsu R, Minagawa J: Energy-dissipative supercomplex of photosystem II associated with LHCSR3 in Chlamydomonas reinhardtii. Proc Natl Acad Sci U S A 2013, 110:10016-10021.

47. Correa-Galvis V, Redekop P, Guan K, Griess A, Truong TB, Wakao S, Niyogi KK, Jahns P: Photosystem II subunit PsbS is involved in the induction of LHCSR protein-dependent energy dissipation in Chlamydomonas reinhardtii. J Biol Chem 2016, 291:17478-17487.

${ }^{* *}$ Although the gene for PsbS is present in C. reinhardtii, the protein function has been a mystery. This paper shows that PsbS protein is transiently expressed at the onset of NPQ and stablizes the accumulation of LHCSR.

48. Li XP, Gilmore AM, Caffarri S, Bassi R, Golan T, Kramer D, Niyogi KK: Regulation of photosynthetic light harvesting involves intrathylakoid lumen $\mathrm{pH}$ sensing by the PsbS protein. J Biol Chem 2004, 279:22866-22874.

49. Niyogi KK, Truong TB: Evolution of flexible non-photochemical quenching mechanisms that regulate light harvesting in oxygenic photosynthesis. Curr Opin Plant Biol 2013, 16:307-314.

50. Gerotto C, Alboresi A, Giacometti GM, Bassi R, Morosinotto T: Coexistence of plant and algal energy dissipation mechanisms in the moss Physcomitrella patens. New Phytol 2012, 196:763-773.

51. Pinnola A, Staleva-Musto H, Capaldi S, Ballottari M, Bassi R, Polívka T: Electron transfer between carotenoid and chlorophyll contributes to quenching in the LHCSR1 protein from Physcomitrella patens. Biochim Biophys Acta 2016, 1857:1870-1878.

52. Pinnola A, Dall'Osto L, Gerotto C, Morosinotto T, Bassi R, Alboresi A: Zeaxanthin binds to light-harvesting complex stress-related protein to enhance nonphotochemical quenching in Physcomitrella patens. Plant Cell 2013, 25:3519-3534.

* Physcomitrella patens exhibits both PsbS-dependent and LHCSR-dependent quenching. This paper shows that the LHCSR-dependent quenching in P. patens is enhanced by binding zeaxanthin under excess light conditions.

53. Gerotto C, Alboresi A, Giacometti GM, Bassi R, Morosinotto T: Role of PSBS and LHCSR in Physcomitrella patens acclimation to high light and low temperature. Plant Cell Environ 2011, 34:922-932. 
54. Leonelli L, Erickson E, Lyska D, Niyogi KK: Transient expression in Nicotiana benthamiana for rapid functional analysis of genes involved in non-photochemical quenching and carotenoid biosynthesis. Plant J 2016, 88:375-386.

55. Pinnola A, Ghin L, Gecchele E, Merlin M, Alboresi A, Avesani L, Pezzotti M, Capaldi S, Cazzaniga S, Bassi R: Heterologous expression of moss light-harvesting complex stress-related 1 (LHCSR1), the chlorophyll a-xanthophyll pigment-protein complex catalyzing non-photochemical quenching, in Nicotiana sp. J Biol Chem 2015, 290:24340-24354.

56. Pinnola A, Cazzaniga S, Alboresi A, Nevo R, Levin-Zaidman S, Reich Z, Bassi R:

Light-harvesting complex stress-related proteins catalyze excess energy dissipation in both photosystems of Physcomitrella patens. Plant Cell 2015, 27:3213-3227.

** This outstanding paper shows the lateral heterogeneity of PSBS- and LHCSR-dependent quenching in Physcomitrella patens, where the former occurs in the PSII-LHCII supercomplex in grana, and the latter occurs in both the PSII-LHCII supercomplex and the PSI-LHCI supercomplex located in the grana margin and stroma lamellae.

57. Gerotto C, Franchin C, Arrigoni G, Morosinotto T: In vivo identification of photosystem II light harvesting complexes interacting with PHOTOSYSTEM II SUBUNIT S. Plant Physiol 2015, 168:1747-1761.

* This paper shows data suggesting the association of PsbS with moderately bound LHCII in PSII-LHCII supercomplex in Physcomitrella patens.

58. Qiu YL, Li L, Wang B, Chen Z, Knoop V, Groth-Malonek M, Dombrovska O, Lee J, Kent L, Rest J, et al.: The deepest divergences in land plants inferred from phylogenomic evidence. Proc Natl Acad Sci U S A 2006, 103:15511-15516. 


\section{Figure 1}

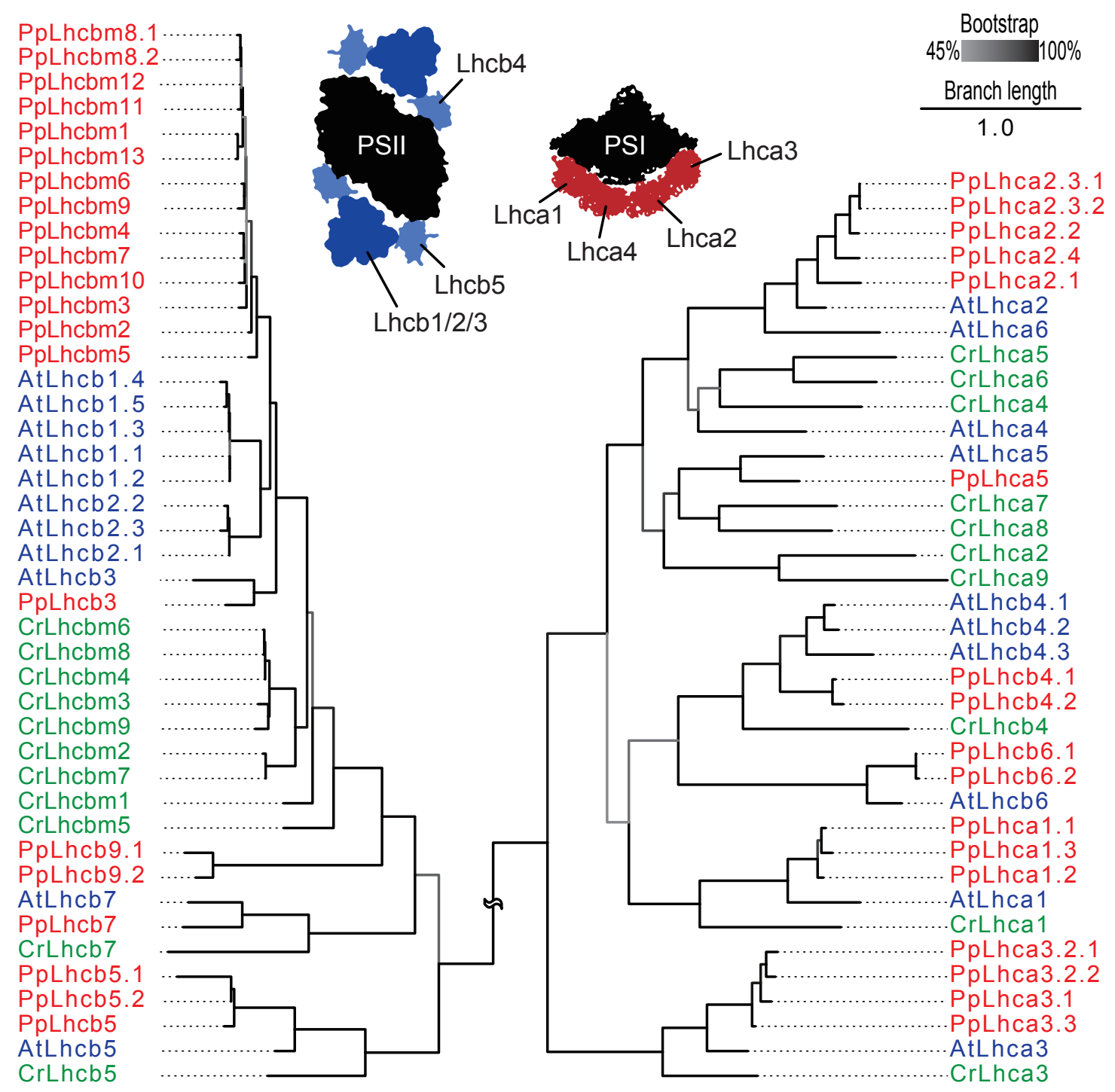


Figure 2

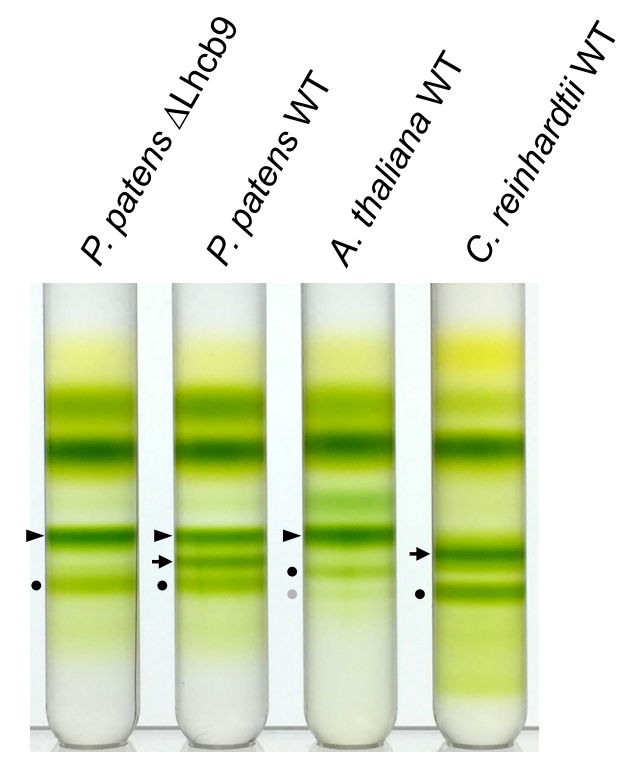




\section{Figure 3}

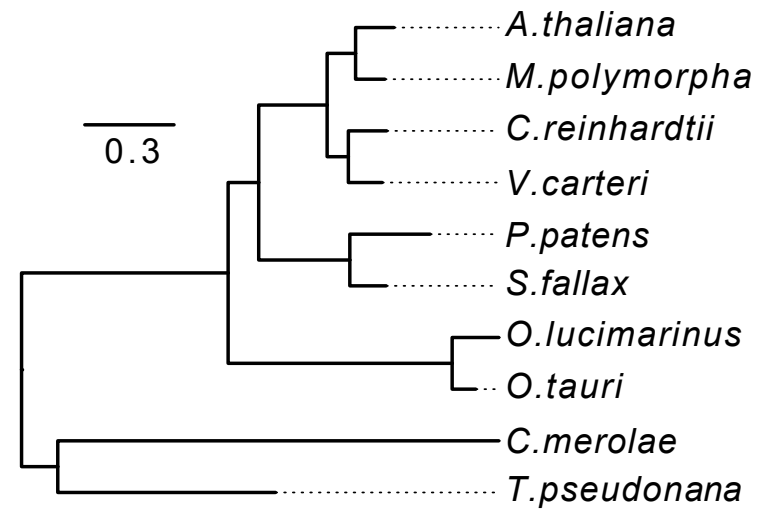

